\title{
Retraction Note to: Toxic potential of different types of sewage sludge as fertiliser in agriculture: ecotoxicological effects on aquatic and soil indicator species
}

\author{
Nadja Rastetter ${ }^{1}$ - Almut Gerhardt ${ }^{1}$
}

Published online: 5 March 2016

(C) Springer-Verlag Berlin Heidelberg 2016

Retraction Note to: J Soils Sediments (2015) 15:565-577

DOI 10.1007/s11368-014-1031-0

This paper has been retracted by the authors on the grounds of honest error. A few calculations were based on analytic values that were incorrectly labeled. The new corrected values have implications for the discussion and conclusion section of the paper. The article will be corrected and resubmitted for peer review. The authors would like to apologize for any inconvenience this may have caused to the readers.

The online version of the original article can be found at http://dx.doi.org/ 10.1007/s11368-014-1031-0.

\footnotetext{
Nadja Rastetter

n_rastetter@web.de

1 LimCo International GmbH, Technology Center Konstanz, Blarerstr. 56, 78462 Konstanz, Germany
} 